\title{
(6) OPEN ACCESS \\ Endovascular recanalization for chronic symptomatic middle cerebral artery total occlusion
}

CASE REPORT

\author{
Ning Ma, ${ }^{1}$ Da-Peng Mo, ${ }^{1}$ Feng Gao, ${ }^{1}$ Zhong-Rong Miao ${ }^{1,2}$
}

\begin{abstract}
${ }^{1}$ Department of Interventional Neuroradiology, Beijing Tiantan Hospital, Capital Medical University, Beijing, China ${ }^{2}$ Department of Neurosurgery, Xuanwu Hospital, Capital Medical University, Beijing, China
\end{abstract}

Correspondence to Dr Z-R Miao, Department of Interventional Neuroradiology, Beijing Tiantan Hospital, Capital Medical University, Beijing 100050, China; zhongrongm@163.com

Received 9 February 2012 Revised 16 March 2012 Accepted 18 March 2012 Published Online First 11 April 2012

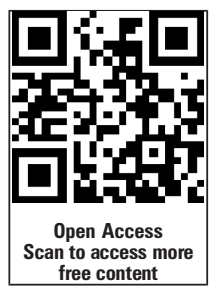

\section{ABSTRACT}

The optimal treatment of chronic middle cerebral artery (MCA) occlusion is unclear. Angioplasty and stenting may be an alternative treatment for patients with recurrent ischemic symptoms despite medical therapy. Two patients with chronic right MCA occlusion successfully treated with stenting are reported, together with their long term follow-up to illustrate the feasibility of endovascular recanalization. One patient remained asymptomatic at the 29-month follow-up. Another patient developed symptomatic in-stent restenosis at 12 months which resolved after repeat angioplasty. Further larger scale pilot studies are needed to determine the efficacy and long term outcome of this treatment.

\section{INTRODUCTION}

A small subset of patients with chronic total occlusion of the middle cerebral artery (MCA) are refractory to medical treatment due to inadequate collateral circulation. ${ }^{1}$ Surgical revascularization with extracranial to intracranial bypass has been the mainstay of treatment but its efficacy remains controversial. ${ }^{2}$ Development of new endovascular devices and improved operator experience have rendered angioplasty and stenting a potential treatment option for such patients. In these case reports, we illustrate the feasibility of elective (at least 1 month after the ischemic event) endovascular recanalization for symptomatic MCA occlusion by angioplasty and stenting, and the long term outcome.

\section{CASE PRESENTATION Case No 1}

A 43-year-old male smoker with hypertension and hyperlipidemia presented with a 2-month history of recurrent transient ischemic attacks (TIA) of left-sided weakness. Brain MRI showed unremarkable right MCA territory. Angiogram demonstrated total occlusion of the right MCA M1 segment with Thrombolysis in Cerebral Ischemia (TICI) grade 0 (figure 1A). Pial collaterals extending from the right anterior cerebral artery (ACA) (figure 1A) and posterior cerebral artery to the right MCA territory were evident. The risks and benefits of conservative medical treatment, extracranial to intracranial bypass and endovascular therapy were discussed with the patient and his families. In light of his recurrent symptoms despite medical therapy with antiplatelet agents, endovascular recanalization of the right MCA was planned. Under general anesthesia, the lesion was traversed with a coaxial assembly of Agility soft microguidewire (Cordis Endovascular, Miami Lakes, Florida, USA) and Prowler 14 microcatheter (Cordis Endovascular, Miami Lakes, Florida, USA) (figure 1B). The microguidewire was then withdrawn, the patency of the lumen distal to the lesion confirmed through the microcatheter (figure 1C) and a Transcend microguidewire (Boston Scientific, Fremont, California, USA) was exchanged. The diameter of the proximal segment of the right MCA adjacent to the lesion was approximately $2.5 \mathrm{~mm}$ and lesion length was $6 \mathrm{~mm}$ by visual estimation, compared with the 6 French guide catheter (Boston Scientific, Natick, Massachusetts, USA). After withdrawing the microcatheter, a $2.5 \times 8 \mathrm{~mm}$ Apollo balloon expandable stent (MicroPort Medical, Shanghai, China) was advanced over the microguidewire and deployed at the lesion. Subsequent angiogram demonstrated resolution of the occlusion and good antegrade perfusion to the right MCA territory (TICI grade 3) (figure 1D). Postoperatively, blood pressure was maintained at 100-120/60-80 mm $\mathrm{Hg}$ to minimize the risk of reperfusion injury. The patient displayed no periprocedural neurological complications verified independently by a neurologist. At his 29-month follow-up, the patient remained asymptomatic on treatment with antiplatelet agent and risk factor control.

\section{Case No 2}

A 66-year-old male smoker with diabetes mellitus and hypertension presented with a 2-month history of TIA of left-sided weakness, similar to case No 1. Brain MRI showed multiple small infarctions in the right MCA territory. Angiogram demonstrated total occlusion of the right MCA M1 segment with TICI grade 0 , and $70 \%$ stenosis of the right ACA ostium (figure 2A). Pial collaterals extending from the right ACA (figure 2B) and posterior cerebral artery contributed to the supply of the right MCA territory. Under general anesthesia, the lesion was traversed with an assembly of Agility soft microguidewire and Prowler 14 microcatheter, which was then exchanged with a Transcend microguidewire. The diameter of the C7 segment of the right internal carotid artery adjacent to the lesion was visually estimated to be $3 \mathrm{~mm}$ and lesion length $8 \mathrm{~mm}$. The occlusion was predilated with a Maverick $2 \times 9 \mathrm{~mm}$ balloon angioplasty (Boston Scientific, Natick, Massachusetts, USA) (figure 2C), followed by deployment of a $2.5 \times 9 \mathrm{~mm}$ Wingspan stent (Boston Scientific, Natick, Massachusetts, USA) covering the entire lesion site (figure 2D). Subsequent angiogram demonstrated complete recanalization of the right 
Figure 1 (A) Angiogram demonstrated total occlusion of the right middle cerebral artery (MCA) M1 segment (arrowhead), and pial collaterals extending from the right anterior cerebral artery territory (arrows). (B) The lesion was traversed with an Agility soft microguidewire (arrowhead). (C) Angiogram through the microcatheter confirming that the tip of the microcatheter was in the distal MCA. (D) Post stenting angiogram showed resolution of the occlusion and good antegrade perfusion to the right MCA territory.
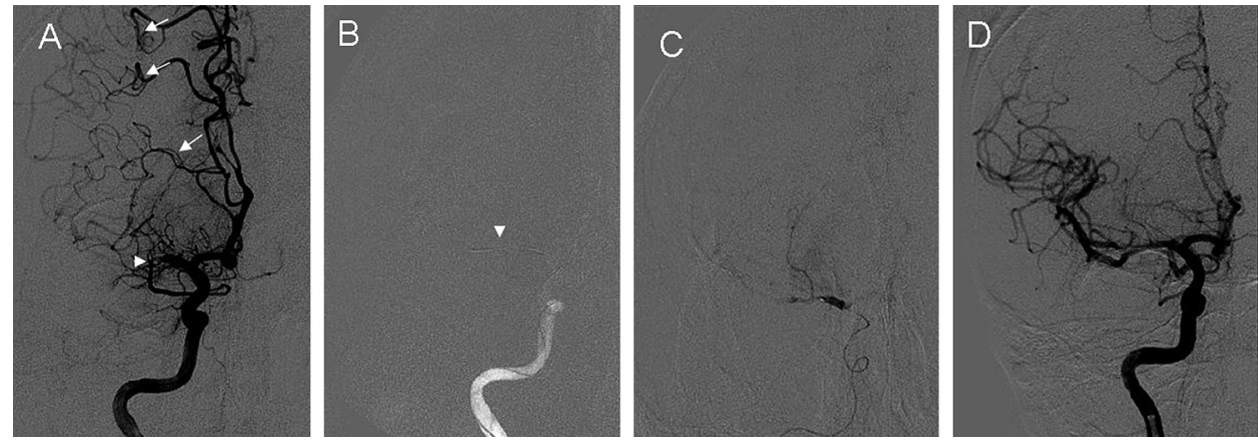

MCA with good antegrade perfusion to the right MCA territory (TICI grade 3) (figure 2E). No perioperative complications were observed. Twelve months later, there was recurrence of TIA of left-sided weakness while on medical treatment. After discussing with the patient and his relatives, repeat endovascular treatment was planned. An $80 \%$ in-stent restenosis (figure $2 \mathrm{~F}$ ) demonstrated on angiogram was successfully treated with Maverick $2 \times 9 \mathrm{~mm}$ balloon angioplasty without complications (figure $2 \mathrm{G}, \mathrm{H}$ ). At his next 12-month follow-up, the patient remained asymptomatic on medical treatment.

\section{DISCUSSION}

Chronic MCA occlusion as a cause of hemodynamic ischemic stroke is not a prominent clinical issue worldwide. ${ }^{1}$ While acute occlusion of the MCA is commonly caused by an embolic thrombus, ${ }^{34}$ atherosclerotic occlusion of the intracranial arteries is more prevalent in Asians. Patients with chronic proximal MCA occlusion may have minor or no stroke because of well developed collaterals. Conversely, the prognosis for those with hemodynamic impairment is poorer. ${ }^{1}$ Chronic occlusive lesion does not seem to represent a significant embolic source but threatens the patient with hemodynamic ischemia and infarct. In addition, patients with MCA occlusion may have disabling cognitive impairment, especially for those with bilateral diseases. ${ }^{5}$ For our two patients, the etiology of their recurrent TIAs was likely to be hemodynamic impairment from total MCA occlusion, arising from atherosclerotic stenosis and superimposed thrombosis based on angiographic findings. The optimal management of them was controversial. ${ }^{2}$

Direct stenting of MCA occlusion has been used for acute stroke but it is rarely performed electively in the chronic phase. ${ }^{6}$ Our cases showed direct stenting without intra-arterial thrombolysis for symptomatic chronic MCA occlusion is feasible. From our experience, the Gateway balloon catheter and the Wingspan stent are more suited for patients with tortuous vascular access, especially at C4 segment, as these afford better
Figure 2 (A) Angiogram demonstrated total occlusion of the right middle cerebral artery (MCA) M1 segment (arrowhead) and 70\% stenosis of the right anterior cerebral artery (ACA) A1 segment (arrow). (B) Pial collaterals extending from the right ACA territory (arrow). (C) The antegrade flow partially improved after predilatation. (D) Stenting deployment (arrowhead). (E) Post stenting angiogram showed good antegrade flow to the right MCA territory. (F) Twelve-month follow-up angiogram demonstrated $80 \%$ in-stent restenosis. (G) Microguidewire was placed in the right ACA to avoid the 'snow ploughing effect' during right MCA angioplasty occluding the right ACA ostium. (H). Post angioplasty angiogram showed resolution of the in-stent stenosis.
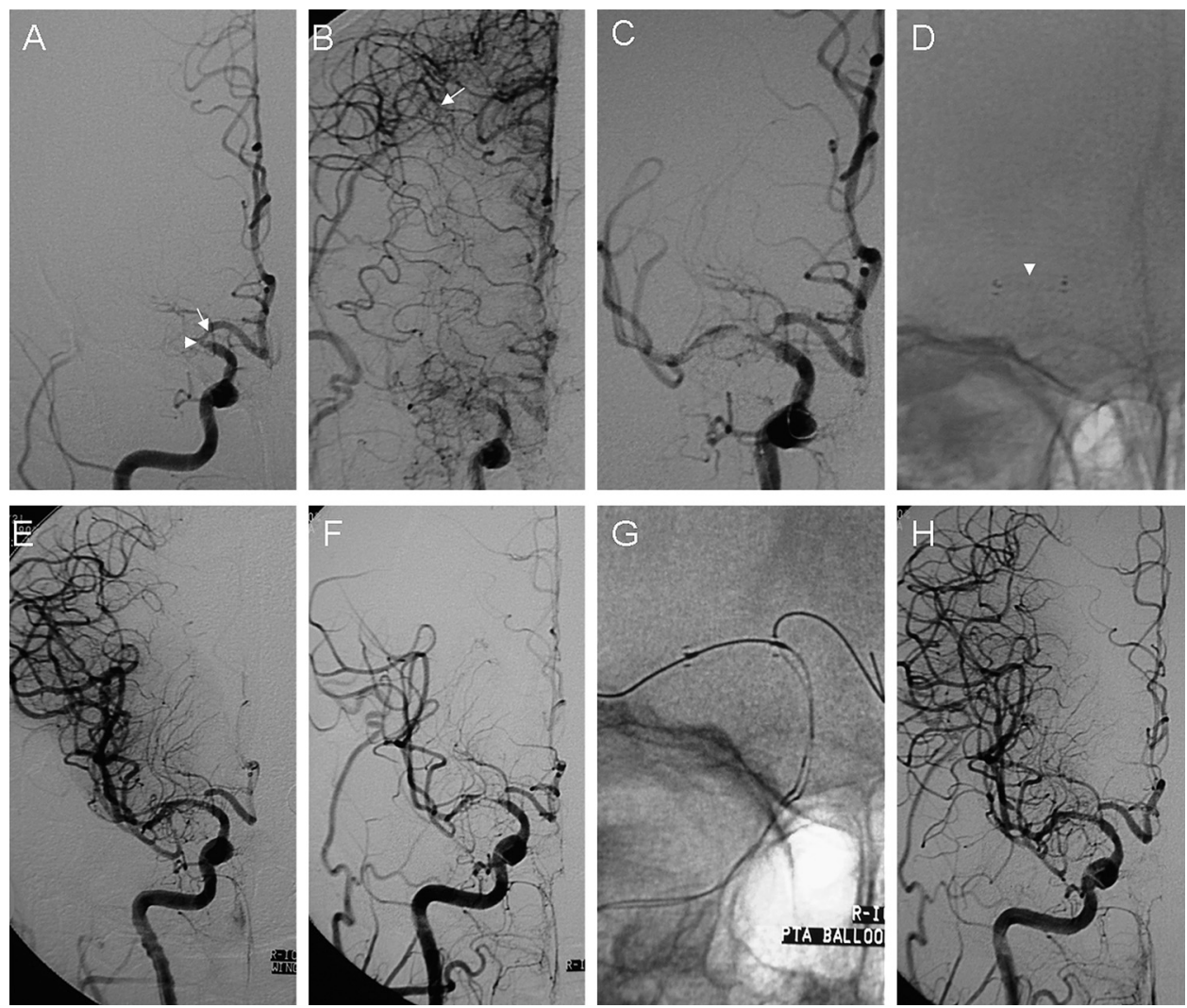
flexibility in traversing the curvature. The Apollo stent is more rigid compared with the Gateway-Wingspan system but it is preferred for patients with a smoother access path as delivery of the balloon expandable stent does not require exchanging and less procedural time was needed. Our immediate technical success rate was $100 \%(2 / 2)$, better than the 33\% (1/3) reported in a previous study which used angioplasty alone. ${ }^{7}$ Although no periprocedural complications occurred in our patients, vessel perforation from microguidewire manipulation or balloon angioplasty was a potentially serious complication. Great care must be taken in monitoring the movement of the microguidewire tip to ensure its position within the true lumen. In the event of dissection, stenting may help to minimize the intimal flaps and hence is superior to angioplasty alone. Hyperperfusion hemorrhage into the recanalized brain parenchyma is another major potential complication, and tight control of blood pressure is crucial postoperatively. During follow-up, one of our two patients developed symptomatic in-stent restenosis which required re-angioplasty. The long term outcome of recanalization of chronic total occlusion of the MCA remains uncertain.

\section{CONCLUSION}

Direct stenting of chronic total occlusion of the MCA is technically feasible but not without the potential for major complications. Further studies are needed to determine its efficacy in carefully selected patients with medically refractory cerebral ischemia due to MCA occlusion.

Acknowledgments The authors thank Dr Edward Ho Chung Wong, MD, at the Department of Medicine and Therapeutics, Chinese University of Hong Kong, Hong Kong, China, for his careful editing of the revision of this article.
Contributors Study concept and design: Z-RM; cases data collection: NM, D-PM and FG; analysis and interpretation of the data: Z-RM and NM; drafting of the manuscript: Z-RM and NM; study supervision: Z-RM.

\section{Competing interests None.}

Patient consent Obtained.

Ethics approval The study was approved by the ethics committee of Beijing Tiantan Hospital, Xuanwu Hospital.

Provenance and peer review Not commissioned; externally peer reviewed.

Open Access This is an Open Access article distributed in accordance with the Creative Commons Attribution Non Commercial (CC BY-NC 3.0) license, which permits others to distribute, remix, adapt, build upon this work non-commercially, and license their derivative works on different terms, provided the original work is properly cited and the use is non-commercial. See: http://creativecommons.org/ licenses/by-nc/3.0/

\section{REFERENCES}

1. Tanaka M, Shimosegawa E, Kajimoto K, et al. Chronic middle cerebral artery occlusion: a hemodynamic and metabolic study with positron-emission tomography. AJNR Am J Neuroradiol 2008;29:1841-6.

2. Bambakidis NC, Chowdhry SA. Cerebral revascularization for ischemic disease in the 21st century. J Neurointerv Surg 2010:2:229-36.

3. Zanette EM, Roberti C, Mancini G, et al. Spontaneous middle cerebral artery reperfusion in ischemic stroke: a follow-up study with transcranial Doppler. Stroke 1995:26:430-3

4. Lhermitte F, Gautier JC, Derouesne C. Nature of occlusions of the middle cerebral artery. Neurology 1970;20:82-8.

5. Wong KSL, Caplan LR, Kim JS. Stroke mechanism. In: Kim JS, Caplan LR, Wong KSL, et al, eds. Intracranial atherosclerosis. Chichester: Blackwell Publishing Ltd, 2008:57-68.

6. Brekenfeld C, Schroth G, Mattle HP, et al. Stent placement in acute cerebral artery occlusion: use of a self-expandable intracranial stent for acute stroke treatment. Stroke 2009:40:847-52.

7. Mori T, Mori K, Fukuoka M, et al. Percutaneous transluminal angioplasty for total occlusion of middle cerebral arteries. Neuroradiology 1997;39:71-_ 